\title{
Integrated Effect of Inorganic and Biofertilizers on Macro and Micro Nutrient Uptake in Strawberry (Fragaria ananassa, Duch)
}

\author{
Zahoor Ahmad Baba ${ }^{1}$, T.A. Sheikh ${ }^{2}$, Amir Hassan $^{3}$, Inayat Mustafa ${ }^{1}$, \\ Tabinda Seher ${ }^{1}$, Gowhar Hussain ${ }^{1}$ and Basharat Hamid ${ }^{1}$
}

\author{
${ }^{1}$ Biofertilizer Research Laboratory, ${ }^{2}$ Division of Agronomy, ${ }^{3}$ Division of Soil Science \\ Faculty of Agriculture, Wadura, Sopore, 193201, India \\ Sher-e-Kashmir University of Agricultural Sciences and Technology of Kashmir, India
}

*Corresponding author

\section{A B S T R A C T}

\begin{tabular}{|l|}
\hline Ke y w or d s \\
Biofertilizers, \\
Inorganic fertilizers, \\
Nutrient uptake, \\
Strawberry \\
\hline Article Info \\
\hline $\begin{array}{l}\text { Accepted: } \\
\text { 20 February } 2018 \\
\text { Available Online: } \\
\text { 10 March } 2018\end{array}$ \\
\hline
\end{tabular}

The present investigation was carried out at the experimental field of Sher-e-Kashmir University of Agricultural Sciences and Technology of Kashmir during the years 2014-15 and 2015-16 to study the combined effect of nitrogen $\left(0,75,150 \mathrm{~kg} \mathrm{ha}^{-1}\right)$, phosphorus $(0$, 50, 100kg ha ${ }^{-1}$ ) and biofertilizers (no inoculants, Azotobacter sp., Bacillus sp., Pseudomonas sp. and Glomus sp.) on macro and micro nutrient uptake in strawberry. The experiment was laid out in completely randomised design (factorial) with 45 treatment combinations and 5 replications. Treatment (T42) recorded significantly higher nitrogen uptake of $0.55 \mathrm{~g}$ and $0.69 \mathrm{~g}$ in $2014-15$ and $2015-16$ respectively. Higher value of phosphorus uptake $(0.11 \mathrm{~g})$ was recorded under the treatments T41, T42, T44 and T45 during the first year while as T41, T42 and T45 recorded significantly higher value of $0.13 \mathrm{~g}$ during the second year. The treatment (T44) recorded significantly higher potassium uptake to the tune of $0.68 \mathrm{~g}$ and $0.78 \mathrm{~g}$ in 2014-15 and 2015-16 respectively. Significantly higher uptake of zinc (11.52 ppm and $12.25 \mathrm{ppm})$, copper (4.82 ppm and $5.30 \mathrm{ppm}$ ), manganese (16.86 ppm and $18.40 \mathrm{ppm})$ and iron (70.93 and $76.60 \mathrm{ppm})$ during both the years of experimentation was observed under the treatment (T45).

\section{Introduction}

Strawberry (Fragaria $\mathrm{x}$ ananassa Duch) is a herbaceous perennial member of the family Rosaceae. Its fruit is highly favoured for its aroma, deliciousness and refreshing quality. In India it is being widely cultivated in the states of Punjab, Haryana, Maharashtra, Himachal Pradesh, Jammu and Kashmir besides some hilly regions of Uttar Pradesh with Maharashtra as a leading state in its production. The importance of soil microorganisms for sustenance of all other life forms needs no emphasis. Microbes are the basis of the biosphere. Soil organisms act as primary driving agents of nutrient cycling, regulating the dynamics of soil organic matter, soil carbon sequestration and greenhouse gas emissions, modifying soil structure and water regimes, enhancing the 
amount of nutrient acquisition by vegetation, conferring stress tolerance, resisting pathogens and improving plant health. Microorganisms play a vital role in the availability of nutrients in soils by their involvement at different stages of organic matter decomposition. They are the sources of most antibiotics and some other drugs and industrial enzymes. More than 90 per cent of the plant's genetic biodiversity is resident in soils.

Rhizosphere is the area of intense microbiological activity and is a highly favourable habitat for the proliferation and metabolism of numerous types of microorganisms. Micro-organisms growing under the influence of roots are often qualitatively and quantitatively different from those inhabiting remote from this influence in the soil environment.

The present investigation was carried out to study the influence of locally isolated, identified and screened rhizosphere microorganisms in combination with inorganic fertilizers on macro and micronutrient uptake in Strawberry.

\section{Materials and Methods}

Strawberry (Fragaria x ananassa, Duch) var. Senga Sangana was grown in the pots in the polyhouse, in Sher-e-Kashmir University of Agricultural Sciences and Technology of Kashmir (SKUAST-K), India during the years 2015-2016.

The soil for filling the pots was collected from the fallow field where no pesticides had been used. The experimental soil was having $\mathrm{pH}$ 6.9, electrical conductivity $0.65 \mathrm{dSm}^{-1}$, organic carbon 0.76 per cent, available nitrogen $99.2 \mathrm{~kg} \mathrm{ha}^{-1}$, phosphorus $10.18 \mathrm{~kg}$ $\mathrm{ha}^{-1}$, potassium $212.16 \mathrm{~kg} \mathrm{ha}^{-1}$, zinc $0.80 \mathrm{ppm}$, copper $0.71 \mathrm{ppm}$, manganese $40.13 \mathrm{ppm}$, iron
$42.91 \mathrm{ppm}$, bacterial population $72 \times 10^{6} \mathrm{CFU}$ $\mathrm{g}^{-1}$ soil, fungal population $45 \times 10^{3} \mathrm{CFU} \mathrm{g} \mathrm{g}^{-1}$ soil and VAM spore population $5 \mathrm{~g}^{-1}$ soil.

The rhizosphere soil samples for the isolation of rhizosphere microflora were obtained from the strawberry experimental field of the Faculty of Agriculture Wadura, SKUAST-K. Azotobacter sp., Bacillus sp. and Pseudomonas $s p$ were isolated by pour plate serial dilution technique (Aneja, 2001) using Ashby's, Pikovaskya and King's B media respectively. Glomus $s p$ spores were isolated by wet sieving and decanting method (Gerdemann and Nicolson, 1963).

The bacterial isolates were identified and screened as per Bergye's Manual of Systematic Bacteriology (Krieg and Holt 1984), while as the VAM isolate was identified and screened as per Phillips and Hayman (1970). These microorganisms were then mass multiplied and used in the experiment laid out in completely randomized design (factorial) with 45 treatment combinations and 5 replications.

Three levels of nitrogen $\left(0,75,150 \mathrm{~kg} \mathrm{ha}^{-1}\right)$ and phosphorus $\left(0,50,100 \mathrm{~kg} \mathrm{ha}^{-1}\right)$ and five levels of microbial inoculants (no inoculant, Azotobacter sp., Bacillus sp., Pseudomonas $s p$., Glomus $s p$ ) were used to study their combined impact on macro and micronutrient uptake by strawberry plants. The treatment combinations are mentioned in Table 1.

\section{Results and Discussion}

\section{Nutrient uptake}

Perusal of data (Table 2) revealed that the combined application of inorganic fertilizers and various bioinoculants significantly influenced the uptake of $\mathrm{N}, \mathrm{P}, \mathrm{K}, \mathrm{Zn}, \mathrm{Cu}, \mathrm{Mn}$ and $\mathrm{Fe}$ in both the years of the experiment. 
In 2014-15 significantly highest nitrogen uptake of $0.55 \mathrm{~g}$ was recorded from the treatment T42 in comparison to control $(0.36$ g).Similarly in 2015-16, significantly highest nitrogen uptake of $0.69 \mathrm{~g}$ was recorded from the same treatment. This might be due to better root development and maximum dry matter production by secretion of various exudates besides biological $\mathrm{N}_{2}$-fixation by Azotobacter inoculation. These findings are in agreement with the results of Rana (2001).

Significantly maximum potassium uptake of $0.68 \mathrm{~g}$ and $0.78 \mathrm{~g}$ was observed during the year 2014-15 and 2015-16 respectively under the treatment T44.The significant enhancement in potassium uptake could be due to the reason that Pseudomonas sp facilitated the availability and uptake of potassium by production of phytohormones and organic acids which increased the solubility of mineral nutrients especially potassium. These findings are supported by the results of Zahir et al (2004) and Esitken (2006).

Significantly higher phosphorus uptake $(0.11 \mathrm{~g})$ was recorded under the treatments like T41, T42, T44, T45 and T41, T42, T45 were at par with respect to the phosphorus uptake $(0.11 \mathrm{~g}$ and $0.13 \mathrm{~g})$ during first and second year of experimentation.

Table.1 Treatment combinations

\begin{tabular}{|c|c|}
\hline$T_{1}=$ Control & $T_{31}=150 \mathrm{~kg} \mathrm{~N} \mathrm{ha}^{-1}$ \\
\hline $\mathbf{T}_{2}=$ Azotobacter Sp. & $\mathrm{T}_{32}=\mathrm{T}_{31}+$ Azotobacter $s p$. \\
\hline $\mathbf{T}_{3}=$ Bacillus $S p$ & $\mathrm{~T}_{33}=\mathrm{T}_{31}+$ Bacillus $s p$ \\
\hline $\mathrm{T}_{4}=$ Pseudomonas $S p$. & $\mathrm{T}_{34}=\mathrm{T}_{31}+$ Pseudomonas $S p$ \\
\hline $\mathrm{T}_{5}=$ Glomus $S p$ & $\mathrm{~T}_{35}=\mathrm{T}_{31}+$ Glomus $s p$ \\
\hline$T_{6}=50 \mathrm{~kg} \mathrm{Pha}^{-1}$ & $\mathrm{~T}_{36}=150 \mathrm{~kg} \mathrm{~N} \mathrm{ha}^{-1}+50 \mathrm{~kg} \mathrm{Pha}^{-1}$ \\
\hline $\mathbf{T}_{7}=\mathbf{T}_{6}+$ Azotobacter $s p$ & $\mathrm{~T}_{37}=\mathrm{T}_{36}+$ Azotobacter $s p$ \\
\hline$T_{8}=T_{6}+$ Bacillus sp. & $\mathrm{T}_{38}=\mathrm{T}_{36}+$ Bacillus $s p$ \\
\hline$T_{9}=T_{6}+$ Pseudomonas sp. & $\mathrm{T}_{39}=\mathrm{T}_{36}+$ Pseudomonas $s p$ \\
\hline $\mathrm{T}_{10}=\mathrm{T}_{6}+$ Glomus sp. & $\mathrm{T}_{24}=\mathrm{T}_{21}+$ Pseudomonas $\mathrm{sp}$ \\
\hline$T_{11}=100 \mathrm{~kg} \mathrm{Pha}^{-1}$ & $\mathrm{~T}_{25}={ }_{21}{ }^{+}$Glomus $\mathrm{sp}$ \\
\hline $\mathrm{T}_{12}=\mathrm{T}_{11}+$ Azotobacter $\mathrm{sp}$. & $\mathrm{T}_{26}=75 \mathrm{~kg} \mathrm{~N} \mathrm{ha}^{-1}+100 \mathrm{~kg} \mathrm{Pha}^{-1}$ \\
\hline$T_{13}=T_{11}+$ Bacillus sp & $\mathrm{T}_{27}=\mathrm{T}_{26}+$ Azotobacter sp. \\
\hline$T_{14}=T_{11}+$ Pseudomonas $\mathrm{sp}$ & $\mathrm{T}_{28}=\mathrm{T}_{26}+$ Bacillus $\mathrm{sp}$ \\
\hline$T_{15}=T_{11}+$ Glomus sp & $\mathrm{T}_{29}=\mathrm{T}_{26}+$ Pseudomonas $\mathrm{sp}$ \\
\hline$T_{16}=75 \mathrm{~kg} \mathrm{~N} \mathrm{ha}^{-1}$ & $\mathrm{~T}_{30}=\mathrm{T}_{26}+$ Glomus $\mathrm{sp}$ \\
\hline $\mathrm{T}_{17}=\mathrm{T}_{16}+$ Azotobacter sp. & $\mathrm{T}_{40}=\mathrm{T}_{36}+$ Glomus $\mathrm{sp}$ \\
\hline $\mathrm{T}_{18}=\mathrm{T}_{16}+$ Bacillus sp & $\mathrm{T}_{41}=150 \mathrm{~kg} \mathrm{~N} \mathrm{ha}^{-1}+100 \mathrm{~kg} \mathrm{Pha}^{-1}$ \\
\hline $\mathrm{T}_{19}=\mathrm{T}_{16}+$ Pseudomonas $\mathrm{sp}$ & $\mathrm{T}_{42}=\mathrm{T}_{41}+$ Azotobacter $\mathrm{sp}$ \\
\hline $\mathrm{T}_{20}=\mathrm{T}_{16}+$ Glomus sp & $\mathrm{T}_{43}=\mathrm{T}_{41}+$ Bacillus $\mathrm{sp}$ \\
\hline$T_{21}=75 \mathrm{~kg} \mathrm{Nha}^{-1}+50 \mathrm{~kg} \mathrm{Pha}^{-1}$ & $\mathrm{~T}_{44}=\mathrm{T}_{41}{ }^{+}$Pseudomonas $\mathrm{sp}$ \\
\hline$T_{22}=T_{21}+$ Azotobacter sp. & $\mathrm{T}_{45}=\mathrm{T}_{41}+$ Glomus $\mathrm{sp}$ \\
\hline$T_{23}=T_{21}+$ Bacillus sp & \\
\hline
\end{tabular}


Table.2 Effect of various microbial inoculants and inorganic fertilizers on uptake of macronutrients

\begin{tabular}{|c|c|c|c|c|c|c|}
\hline \multirow{2}{*}{ Treatments } & \multicolumn{2}{|c|}{ Nitrogen uptake(g) } & \multicolumn{2}{|c|}{ Phosphorus uptake(g) } & \multicolumn{2}{|c|}{ Potassium uptake(g) } \\
\hline & 2014-15 & 2015-16 & 2014-15 & 2015-16 & 2014-15 & 2015-16 \\
\hline $\mathbf{T}_{1}$ & 0.36 & 0.47 & 0.07 & 0.11 & 0.46 & 0.54 \\
\hline $\mathrm{T}_{2}$ & 0.40 & 0.53 & 0.07 & 0.11 & 0.51 & 0.60 \\
\hline $\mathbf{T}_{3}$ & 0.37 & 0.49 & 0.07 & 0.10 & 0.49 & 0.57 \\
\hline $\mathbf{T}_{4}$ & 0.39 & 0.51 & 0.06 & 0.11 & 0.51 & 0.60 \\
\hline $\mathbf{T}_{5}$ & 0.38 & 0.50 & 0.07 & 0.11 & 0.49 & 0.57 \\
\hline$T_{6}$ & 0.38 & 0.50 & 0.07 & 0.10 & 0.49 & 0.57 \\
\hline $\mathrm{T}_{7}$ & 0.41 & 0.53 & 0.07 & 0.09 & 0.51 & 0.60 \\
\hline $\mathbf{T}_{8}$ & 0.40 & 0.52 & 0.07 & 0.09 & 0.51 & 0.60 \\
\hline $\mathbf{T}_{9}$ & 0.40 & 0.53 & 0.07 & 0.09 & 0.52 & 0.61 \\
\hline $\mathbf{T}_{10}$ & 0.40 & 0.52 & 0.21 & 0.10 & 0.51 & 0.60 \\
\hline $\mathbf{T}_{11}$ & 0.42 & 0.53 & 0.08 & 0.10 & 0.52 & 0.61 \\
\hline $\mathrm{T}_{12}$ & 0.42 & 0.54 & 0.07 & 0.09 & 0.60 & 0.61 \\
\hline $\mathbf{T}_{13}$ & 0.40 & 0.53 & 0.08 & 0.10 & 0.60 & 0.61 \\
\hline$T_{14}$ & 0.41 & 0.53 & 0.08 & 0.10 & 0.53 & 0.62 \\
\hline $\mathbf{T}_{15}$ & 0.41 & 0.53 & 0.08 & 0.10 & 0.52 & 0.61 \\
\hline $\mathbf{T}_{16}$ & 0.43 & 0.55 & 0.07 & 0.09 & 0.53 & 0.61 \\
\hline $\mathrm{T}_{17}$ & 0.45 & 0.57 & 0.08 & 0.10 & 0.53 & 0.62 \\
\hline $\mathbf{T}_{18}$ & 0.42 & 0.55 & 0.08 & 0.09 & 0.53 & 0.61 \\
\hline $\mathbf{T}_{19}$ & 0.41 & 0.54 & 0.08 & 0.09 & 0.53 & 0.62 \\
\hline $\mathbf{T}_{20}$ & 0.42 & 0.54 & 0.08 & 0.10 & 0.53 & 0.62 \\
\hline $\mathbf{T}_{21}$ & 0.44 & 0.57 & 0.08 & 0.11 & 0.55 & 0.64 \\
\hline $\mathrm{T}_{22}$ & 0.47 & 0.60 & 0.09 & 0.11 & 0.56 & 0.65 \\
\hline $\mathbf{T}_{23}$ & 0.43 & 0.57 & 0.09 & 0.11 & 0.55 & 0.64 \\
\hline $\mathbf{T}_{24}$ & 0.44 & 0.57 & 0.08 & 0.10 & 0.56 & 0.65 \\
\hline $\mathbf{T}_{25}$ & 0.44 & 0.57 & 0.09 & 0.11 & 0.56 & 0.65 \\
\hline $\mathbf{T}_{26}$ & 0.45 & 0.58 & 0.10 & 0.12 & 0.56 & 0.65 \\
\hline $\mathrm{T}_{27}$ & 0.48 & 0.61 & 0.10 & 0.12 & 0.57 & 0.67 \\
\hline $\mathbf{T}_{28}$ & 0.46 & 0.60 & 0.10 & 0.12 & 0.56 & 0.65 \\
\hline $\mathbf{T}_{29}$ & 0.47 & 0.61 & 0.09 & 0.11 & 0.58 & 0.67 \\
\hline $\mathbf{T}_{30}$ & 0.47 & 0.60 & 0.10 & 0.12 & 0.58 & 0.67 \\
\hline $\mathbf{T}_{31}$ & 0.49 & 0.59 & 0.08 & 0.10 & 0.57 & 0.64 \\
\hline $\mathrm{T}_{32}$ & 0.51 & 0.63 & 0.08 & 0.10 & 0.57 & 0.67 \\
\hline $\mathbf{T}_{33}$ & 0.48 & 0.61 & 0.08 & 0.10 & 0.57 & 0.66 \\
\hline $\mathbf{T}_{34}$ & 0.48 & 0.61 & 0.08 & 0.10 & 0.57 & 0.66 \\
\hline $\mathbf{T}_{35}$ & 0.49 & 0.62 & 0.07 & 0.11 & 0.58 & 0.67 \\
\hline
\end{tabular}




\begin{tabular}{|l|l|l|l|l|l|l|}
\hline $\mathbf{T}_{\mathbf{3 6}}$ & 0.50 & 0.62 & 0.09 & 0.11 & 0.59 & $\mathbf{0 . 6 8}$ \\
\hline $\mathbf{T}_{37}$ & 0.54 & 0.67 & 0.09 & 0.11 & 0.65 & $\mathbf{0 . 7 4}$ \\
\hline $\mathbf{T}_{\mathbf{3 8}}$ & 0.50 & 0.63 & 0.20 & 0.11 & 0.63 & $\mathbf{0 . 7 3}$ \\
\hline $\mathbf{T}_{\mathbf{3 9}}$ & 0.51 & 0.64 & 0.20 & 0.10 & 0.64 & $\mathbf{0 . 7 4}$ \\
\hline $\mathbf{T}_{\mathbf{4 0}}$ & 0.51 & 0.64 & 0.09 & 0.12 & 0.63 & $\mathbf{0 . 7 3}$ \\
\hline $\mathbf{T}_{\mathbf{4 1}}$ & 0.50 & 0.64 & 0.11 & 0.13 & 0.60 & $\mathbf{0 . 6 9}$ \\
\hline $\mathbf{T}_{42}$ & 0.55 & 0.69 & 0.11 & 0.13 & 0.66 & $\mathbf{0 . 7 5}$ \\
\hline $\mathbf{T}_{\mathbf{4 3}}$ & 0.51 & 0.65 & 0.10 & 0.12 & 0.65 & $\mathbf{0 . 7 4}$ \\
\hline $\mathbf{T}_{\mathbf{4 4}}$ & 0.54 & 0.68 & 0.11 & 0.12 & 0.68 & $\mathbf{0 . 7 8}$ \\
\hline $\mathbf{T}_{\mathbf{4 5}}$ & 0.53 & 0.66 & 0.11 & 0.13 & 0.66 & $\mathbf{0 . 7 6}$ \\
\hline $\mathbf{C D}_{\mathbf{( 0 . 0 5 )}}$ & $\mathbf{0 . 0 1}$ & $\mathbf{0 . 0 1}$ & $\mathbf{0 . 0 1}$ & $\mathbf{0 . 0 1}$ & $\mathbf{0 . 0 1}$ & $\mathbf{0 . 0 1}$ \\
\hline
\end{tabular}

Table.3 Effect of various microbial inoculants and inorganic fertilizers on uptake of micronutrient cations

\begin{tabular}{|c|c|c|c|c|c|c|c|c|}
\hline \multirow{2}{*}{ Treatments } & \multicolumn{2}{|c|}{$\begin{array}{l}\text { Zinc } \\
\text { uptake(ppm) }\end{array}$} & \multicolumn{2}{|c|}{$\begin{array}{l}\text { Copper } \\
\text { uptake(ppm) }\end{array}$} & \multicolumn{2}{|c|}{$\begin{array}{l}\text { Manganese } \\
\text { Uptake(ppm) }\end{array}$} & \multicolumn{2}{|c|}{$\begin{array}{l}\text { Iron } \\
\text { uptake(ppm) }\end{array}$} \\
\hline & 2014-15 & 2015-16 & 2014-15 & 2015-16 & 2014-15 & 2015-16 & 2014-15 & 2015-16 \\
\hline $\mathbf{T}_{1}$ & 5.70 & 6.21 & 1.45 & 1.78 & 11.67 & 11.75 & 53.73 & 57.97 \\
\hline $\mathrm{T}_{2}$ & 6.39 & 7.03 & 2.04 & 2.45 & 12.24 & 13.77 & 59.62 & 63.20 \\
\hline $\mathbf{T}_{3}$ & 6.58 & 7.19 & 1.95 & 2.31 & 11.97 & 13.29 & 57.57 & 61.19 \\
\hline $\mathbf{T}_{4}$ & 6.61 & 7.15 & 1.98 & 2.38 & 12.40 & 13.74 & 59.62 & 62.45 \\
\hline $\mathbf{T}_{5}$ & 6.65 & 7.20 & 2.22 & 2.58 & 12.46 & 13.63 & 58.18 & 61.35 \\
\hline$T_{6}$ & 6.37 & 6.91 & 1.80 & 2.15 & 11.74 & 12.99 & 57.74 & 61.58 \\
\hline $\mathrm{T}_{7}$ & 6.99 & 7.67 & 2.21 & 2.53 & 12.87 & 14.24 & 61.67 & 64.80 \\
\hline $\mathbf{T}_{8}$ & 7.17 & 7.74 & 2.14 & 2.51 & 12.97 & 14.34 & 61.59 & 65.02 \\
\hline $\mathbf{T}_{9}$ & 7.24 & 7.82 & 2.18 & 2.56 & 12.83 & 14.19 & 62.32 & 65.50 \\
\hline $\mathbf{T}_{10}$ & 7.44 & 8.02 & 2.50 & 2.88 & 13.52 & 14.91 & 62.37 & 65.70 \\
\hline $\mathbf{T}_{11}$ & 7.17 & 7.54 & 2.05 & 2.42 & 12.76 & 13.92 & 61.47 & 64.35 \\
\hline $\mathrm{T}_{12}$ & 7.30 & 7.88 & 2.34 & 2.72 & 12.93 & 14.31 & 63.60 & 66.95 \\
\hline $\mathbf{T}_{13}$ & 7.82 & 8.41 & 2.61 & 3.00 & 13.14 & 14.52 & 63.93 & 67.30 \\
\hline $\mathbf{T}_{14}$ & 7.84 & 8.50 & 2.61 & 3.01 & 13.17 & 14.57 & 64.01 & 67.39 \\
\hline $\mathbf{T}_{15}$ & 8.07 & 8.68 & 2.82 & 3.22 & 14.23 & 15.64 & 65.14 & 68.56 \\
\hline $\mathbf{T}_{16}$ & 7.27 & 7.84 & 2.05 & 2.42 & 12.60 & 13.95 & 61.15 & 64.59 \\
\hline $\mathrm{T}_{17}$ & 7.94 & 8.54 & 2.37 & 2.75 & 13.07 & 14.45 & 63.20 & 66.38 \\
\hline$T_{18}$ & 8.13 & 8.74 & 2.34 & 2.72 & 13.20 & 14.58 & 63.32 & 66.79 \\
\hline
\end{tabular}




\begin{tabular}{|c|c|c|c|c|c|c|c|c|}
\hline$T_{19}$ & 8.21 & 8.82 & 2.64 & 3.04 & 13.26 & 14.43 & 64.01 & 67.23 \\
\hline $\mathbf{T}_{20}$ & 8.67 & 9.30 & 2.61 & 3.00 & 13.73 & 15.14 & 65.72 & 69.40 \\
\hline $\mathbf{T}_{21}$ & 8.34 & 8.93 & 2.39 & 2.78 & 13.50 & 14.91 & 65.64 & 69.25 \\
\hline $\mathrm{T}_{22}$ & 8.96 & 9.60 & 2.70 & 3.10 & 14.08 & 15.63 & 66.89 & 70.37 \\
\hline $\mathbf{T}_{23}$ & 8.93 & 9.33 & 2.67 & 3.05 & 14.34 & 15.66 & 66.85 & 69.98 \\
\hline $\mathbf{T}_{24}$ & 8.90 & 9.53 & 2.97 & 3.38 & 14.37 & 15.81 & 67.14 & 70.49 \\
\hline $\mathbf{T}_{25}$ & 9.40 & 9.93 & 3.23 & 3.64 & 14.72 & 16.17 & 67.47 & 71.03 \\
\hline $\mathbf{T}_{26}$ & 8.99 & 9.52 & 2.68 & 3.07 & 14.06 & 15.49 & 66.44 & 70.12 \\
\hline $\mathrm{T}_{27}$ & 9.92 & 10.35 & 3.31 & 3.73 & 14.91 & 16.37 & 68.56 & 71.71 \\
\hline $\mathbf{T}_{28}$ & 7.91 & 10.42 & 3.25 & 3.67 & 14.65 & 16.10 & 67.69 & 71.46 \\
\hline $\mathbf{T}_{29}$ & 10.25 & 10.94 & 3.03 & 3.44 & 15.26 & 16.74 & 69.09 & 72.34 \\
\hline $\mathbf{T}_{30}$ & 10.57 & 11.27 & 3.52 & 3.95 & 15.46 & 16.94 & 68.04 & 71.28 \\
\hline $\mathbf{T}_{31}$ & 8.21 & 8.59 & 2.42 & 2.75 & 13.50 & 14.51 & 65.88 & 68.12 \\
\hline $\mathrm{T}_{32}$ & 8.54 & 9.16 & 2.75 & 3.16 & 14.15 & 15.59 & 68.57 & 69.96 \\
\hline $\mathbf{T}_{33}$ & 8.75 & 9.32 & 2.66 & 3.11 & 14.06 & 15.36 & 66.45 & 69.53 \\
\hline $\mathbf{T}_{34}$ & 9.02 & 9.66 & 2.71 & 3.12 & 14.28 & 15.72 & 66.33 & 69.54 \\
\hline $\mathbf{T}_{35}$ & 9.08 & 9.72 & 3.02 & 3.44 & 14.68 & 16.12 & 67.37 & 70.96 \\
\hline $\mathbf{T}_{36}$ & 8.55 & 9.17 & 2.75 & 3.16 & 14.16 & 15.60 & 69.95 & 71.74 \\
\hline $\mathrm{T}_{37}$ & 9.66 & 10.14 & 3.42 & 3.86 & 15.42 & 16.91 & 70.02 & 73.26 \\
\hline $\mathbf{T}_{38}$ & 9.79 & 10.45 & 3.36 & 3.79 & 14.86 & 16.32 & 69.69 & 73.40 \\
\hline $\mathbf{T}_{39}$ & 9.94 & 10.61 & 3.71 & 4.16 & 15.69 & 17.19 & 70.70 & 73.95 \\
\hline $\mathbf{T}_{40}$ & 10.34 & 11.03 & 3.86 & 4.39 & 16.28 & 17.80 & 70.34 & 73.85 \\
\hline $\mathbf{T}_{41}$ & 10.04 & 10.72 & 3.35 & 3.78 & 15.38 & 16.87 & 68.52 & 72.50 \\
\hline $\mathrm{T}_{42}$ & 10.65 & 11.35 & 4.05 & 4.51 & 16.16 & 17.68 & 71.52 & 74.55 \\
\hline $\mathbf{T}_{43}$ & 10.65 & 11.35 & 3.94 & 4.39 & 16.01 & 17.52 & 70.45 & 74.45 \\
\hline $\mathbf{T}_{44}$ & 11.43 & 12.16 & 4.42 & 4.90 & 16.72 & 18.26 & 73.25 & 75.31 \\
\hline $\mathbf{T}_{45}$ & 11.52 & 12.25 & 4.82 & 5.30 & 16.86 & 18.40 & 70.93 & 76.60 \\
\hline CD (0.05) & 0.60 & 0.60 & 0.40 & 0.40 & 1.27 & 1.27 & 4.58 & 4.58 \\
\hline
\end{tabular}

Data presented in Table 2 and 3 represented that during both the years of experimentation treatment T45 significantly influenced the $\mathrm{Zn}, \mathrm{Cu}, \mathrm{Mn}$ and $\mathrm{Fe}$ uptake with maximum uptake of $\mathrm{Zn}(11.52 \mathrm{ppm}$ and $12.25 \mathrm{ppm}), \mathrm{Cu}$ (4.82 ppm and $5.30 \mathrm{ppm}$ ), Mn (16.86 ppm and $18.40 \mathrm{ppm})$ and $\mathrm{Fe}$ (70.93 ppm and 76.60 ppm) respectively. This might be due to the active involvement of Glomus sp.in enhancing the phosphorus uptake besides increasing the root absorption surface area which in turn enhanced the micronutrient uptake. Hughes et al., (1978) and Subramanian et al., (2006) also reported similar findings with arbuscular mycorrhizae

From the present study it can be concluded that there is an ample population of beneficial 
rhizosphere microflora that can be exploited for improving the plant growth and yield. Therefore, the main recommendation from this experiment is that the combined application of $150 \mathrm{~kg} \mathrm{~N} \mathrm{ha}^{-1}$ and $100 \mathrm{~kg} \mathrm{P} \mathrm{ha}^{-1}$ along with Azotobacter sp., Pseudomonas sp. and VAM fungi will go a long way in improving growth, yield and quality of strawberry.

\section{References}

Aneja, K.R. 2001. Experiments in microbiology, plant pathology, tissue culture and mushroom production technology. Third Edition. New Age International Publishers, Pvt. Ltd., New Delhi, pp. 568.

Esitken, A. Pirlak, L., Turan, M. and Sahim, F. 2006. Effect of floral and foliar application of plant growth promoting rhizobacteria (PGPR) on yield, growth and nutrition of sweet cherry. Scientia Horticulturae, 110:324-327.

Gerdemann, I.W. and Nicolson, T.H. 1963. Spores of mycorrhizal endogone species extracted from soil by wet sieving and decanting. Trans Britanica. Mycological Society, 46: 235-244.

Gomez, A.A. and Gomez, K.A. 1984 Statistical Procedures for Agricultural Research. John Wiley and Sons, Inc., New York, pp 680.

Hughes, M., Martin, L.W. and Breen, P.J. 1978. Mycorrhizal influence on the nutrition of strawberries. Journal of American Society for Horticultural
Sciences 103(2): 179-181.

Jackson, M.L. 1973. Soil Chemical Analysis. Prentice Hall of India, Pvt. Ltd., New Delhi, pp 498.

Krieg, R.N. and Holt, G.J. 1984. Bergey's Manual of Systemic Bacteriology. Volume I. Williams and Wilkins, 428 East Preston Street Baltimore, MD 21202, USA, pp 837.

Phillips, J. and Hayman, D. 1970. Improved procedures for clearing roots and staining parasitic and vesicular arbuscular mycorrhizal fungi for rapid assessment of infection. Trans Britanica Mycological Society 55: 158-161.

Rana, R.K. 2001. Studies on the influence of nitrogen fixers and plant bioregulators on growth, yield and fruit quality of strawberry cv. Chandler. Ph.D thesis submitted to Dr. Y.S. Parmar University of Horticulture and Forestry, Nauni, Solan, India, pp 41138.

Subramanian, K.S., Santhana Krishnan, P. and Balasubramanian, P. 2006. Response of field grown tomato plants to arbuscular mycorrhizal fungal colonization under varying intensities of drought stress. Scientia Horticulturae, 107: 245-253.

Zahir, A., Zahir, M.A. and William, T.F.J. 2004. Plant growth promoting rhizobacteria. Applications and perspectives in agriculture. Advances in Agronomy 81: 97-149.

\section{How to cite this article:}

Zahoor Ahmad Baba, T.A. Sheikh, Amir Hassan, Inayat Mustafa, Tabinda Seher, Gowhar Hussain and Basharat Hamid. 2018. Integrated Effect of Inorganic and Biofertilizers on Macro and Micro Nutrient Uptake in Strawberry (Fragaria ananassa Duch). Int.J.Curr.Microbiol.App.Sci. 7(03): 2146-2152. doi: https://doi.org/10.20546/ijcmas.2018.703.252 